\title{
Skin Maceration
}

National Cancer Institute

\section{Source}

National Cancer Institute. Skin Maceration. NCI Thesaurus. Code C83472.

Softening and whitening of the skin due to excessive moisture. 